\title{
Study on the Vacuum-Degassing Technology of the Oil-Paper Insulated
}

\section{Transformer}

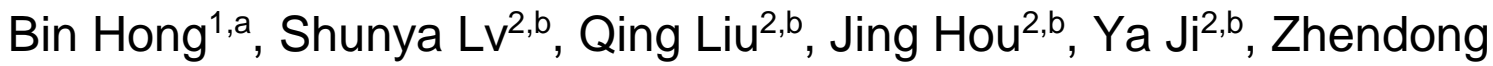 \\ $\mathrm{Gao}^{2, \mathrm{~b}}$, Jianye $\mathrm{Gao}^{2, \mathrm{~b}}$, Ju Hu $\mathrm{H}^{2, \mathrm{~b}}$ \\ ${ }^{1}$ Internal Combustion Engine Research Institute,Tianjin University, Tianjin 300072, China \\ ${ }^{2}$ Tianbo Science \& Technology Co. Ltd., Tianjin 300072, China \\ atianbohb@126.com, btianbojy905@126.com
}

\begin{abstract}
Keywords: the oil-paper insulated transformer, vacuum-degassing technology, the pouring process, air bubble

Abstract:The 3D model of the oil-paper insulated transformer was constructed by SOLIDWORKS and simulated by Moldflow to optimize its vacuum-degassing technology. The number of the inside oil papers, the space of the adjacent two oil papers, the number and its distribution of the setting oil inlets are discussed in detail and the variation of the relevant formed air bubbles are analyzed. It reveals that the pouring process and the residual air bubbles are seriously influenced by the number and the space of the oil papers. In order to exhaust the inside air, the reserved oil inlets should be set symmetrically on the top of the transformer. Furthermore, the formation mechanism of the residual air bubbles is proposed.
\end{abstract}

\section{Introduction}

Power transformers are the most critical and expensive equipment in the field of transmission and distribution of electric energy [1,2]. It is one of the most important pieces of equipment in electrical systems [3].

The transformer with an oil-paper structure inside is the widely used as the normal power insulation. To ensure the reliability of oil-immersed power transformers in long-term operation, many researchers have carried out a series of related investigations subsequently $[4,5,6]$. Investigations on electrical, thermal, or electrical thermal aging of oil-paper insulation have attracted lots of interests of researchers for more than decades of years $[7,8]$.

One among the most dangerous effects that causes failures of power transformers is the bubble effect, during which a violent release of water vapor from cellulose insulation takes place after exceeding a critical temperature and a critical moisture content. The bubble effect results in a rapid increase of tank pressure, which may thereafter cause an explosion and a fire event [9].

In order to avoid or mitigate such failure risk, research on the vacuum-degassing technology is essential for the oil-paper insulated transformer. A proper pouring technology can directly influence the degassing effects of the air bubbles and moisture inside the transformer. Therefore, the 3D model of the oil-paper insulated transformer was constructed by SOLIDWORKS and simulated by Moldflow to optimize its vacuum-degassing technology. The pouring process influenced by the space and quality of the oil papers, the number and its distribution of the oil inlets were discussed. Moreover, the formation mechanism of the residual air bubbles is proposed properly. 


\section{Construction of 3D model and its simulating process}

According to the specific vacuum-degassing structure of the oil-paper insulated transformer, its 3D fluid model was constructed by SOLIDWORKS as shown in Fig.1. Obviously, the transformer includes the oil inlets, the oil papers and the transformer oil. Considering the variations of the spacing between two oil-paper layers, the number of the oil paper, the number and their distribution of the reserved inlets, the 3D fluid models are modified separately which can be found in the result and discussion part in detail.

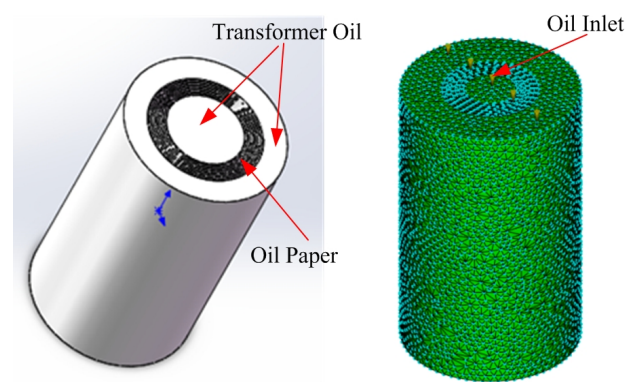

Fig.1 The 3D fluid model of the oil-paper insulated transformer

Based on the constructed 3D fluid model, available material was chosen and the vacuum pouring technology was determined during the Moldflow simulating process. With the simulation and analysis of the fluid model, the spacing and the number of the oil papers, the number and their distribution of the oil inlets are discussed during the vacuum degassing process.

\section{Results and discussion}

\section{The pouring process influenced by the space of the adjacent oil papers}

With the pouring pressure of $5000 \mathrm{~Pa}$, the vacuum degassing process was investigated when the spaces between the two oil papers are mainly set as $0.5 \mathrm{~mm}, 0.8 \mathrm{~mm}$ and $1.0 \mathrm{~mm}$. the simulating results are shown in Fig.2 and Fig.3. The pouring time of the transformer with diverse paper spaces is displayed in Fig.2. In this figure, it can be observed that the oil firstly flow down along the center part, then flow up to fill the outside part and soak into the oil papers. Compared with Fig.2 (a), the pouring time about the center part is quite shorten when the oil-paper space is enlarged from 0.5 $\mathrm{mm}$ to $0.8 \mathrm{~mm}$, and the soaking speed in the oil paper spaces is rapidly increased. It reveals that the bigger oil-paper is beneficial for the soaking process in the oil papers and the pouring time is extended due to the larger volume between the whole papers. When the oil-paper space is continuously enlarged to $1.0 \mathrm{~mm}$, it can be found that the pouring time is obviously longer compared with Fig.2. (b). Therefore, the space between the oil papers is essential to the oil pouring process, and seriously affected the pouring quality and its reliability of the transformer. 


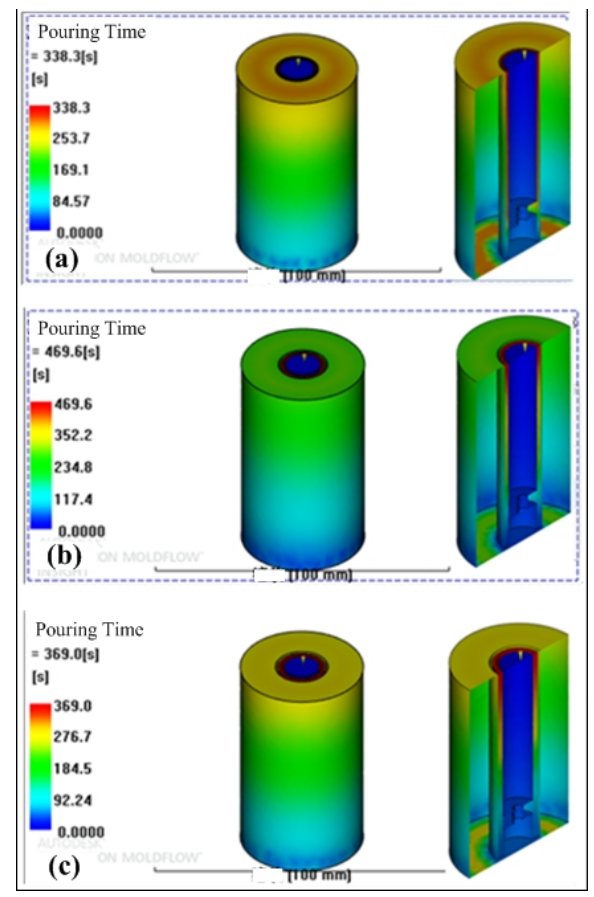

(a) $0.5 \mathrm{~mm}$; (b) $0.8 \mathrm{~mm}$; (c) $1.0 \mathrm{~mm}$

Fig. 2 The simulated pouring time of the transformer with diverse oil-paper spaces

The distribution of the residual air bubbles inside the transformer according to the different spaces between the adjacent two oil papers is exhibited in Fig.3. It can be seen that the residual air bubbles mainly concentrate on the top and the bottle of the simulated model. Moreover, the distribution, the number and the size of the residual air bubbles are rarely influenced by the oil-paper space.

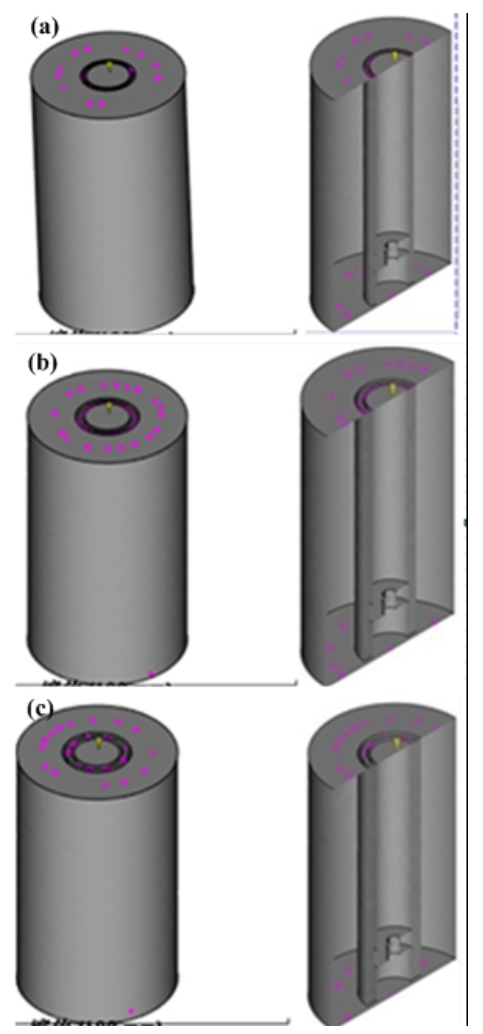

Fig.3 The distribution of residual air bubbles formed on the transformer with diverse oil-paper spaces 


\section{The pouring process influenced by the oil-paper layers}

The transformer with three oil-paper layers is simulated as shown in Fig.3 when a pouring inlet is set on the top of the center part and the space between the adjacent two papers is $0.5 \mathrm{~mm}$ under a pouring pressure of $5000 \mathrm{~Pa}$. In this case, although oil can still flow into the center part and the outside part of the obtained model feasibly, the soaking process is quite difficult as there is large air void in the oil-paper spaces. Therefore, as the oil-paper layers increased, larger pouring pressure is needed to guarantee the oil can soak into the oil-paper space successfully.

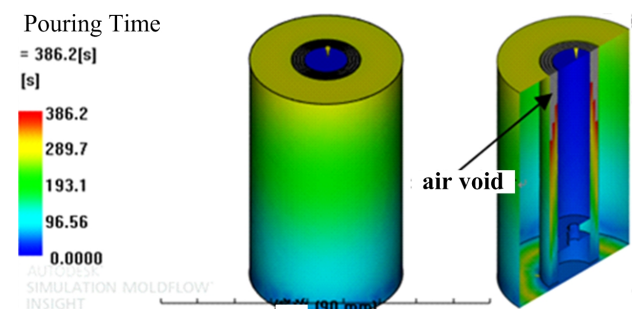

Fig.4 The simulated pouring time of the transformer with three oil-paper layers under a pouring pressure of $500 \mathrm{~Pa}$

According to the above analysis, the pouring pressure is raised to $0.05 \mathrm{MPa}$ when the oil-paper layer of the transformer is increased and the relevant simulating results are presented in Fig.5 and Fig.6. In Fig.5, it can be observed that the pouring time is extended but the pouring speed filling the center part is rarely influenced when the transformers contain three paper layers and five paper layers, respectively.

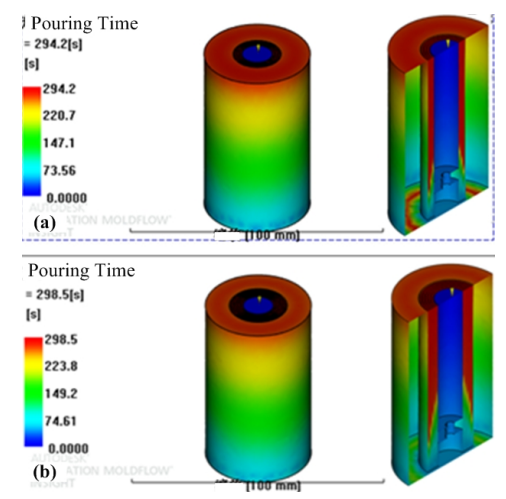

(a) three oil-paper layers, (b) five oil-paper layers

Fig.5 The simulated pouring time of the transformers simulated with a pouring pressure of 0.05 $\mathrm{MPa}$

The relevant distribution of the residual air bubbles can be seen in Fig.6. Obviously, the air bubbles still mainly concentrate on the top and the bottle of the transformer. But as the oil-paper layer increased, more air bubbles is formed on the outside surface of the model, especially the air bubbles generated on the top of the oil-paper spaces. Therefore, the pouring technology is seriously affected by the oil-paper layers. 


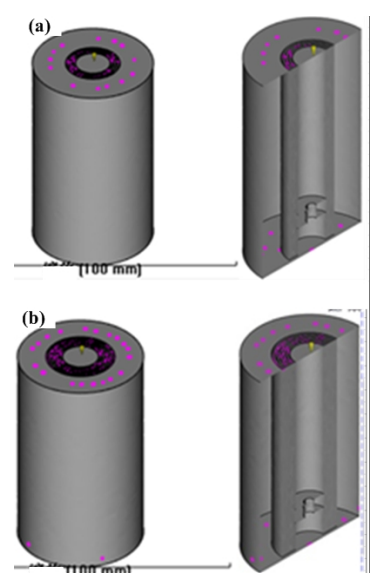

(a) three oil-paper layers, (b) five oil-paper layers

Fig. 6 The distribution of residual air bubbles formed on transformer with a pouring pressure of 0.05 $\mathrm{MPa}$

\section{The pouring process influenced by the reserved oil inlets}

The number and distribution of the reserved pouring inlets are important to the vacuum degassing effect of transformer. In this part, the simulating condition was adjusted as the pouring pressure was $0.5 \mathrm{MPa}$, the oil-paper space was $0.5 \mathrm{~mm}$ and the transformer included five paper layers.

The inlets set on the top of the center part and outside part was simulated as shown in Fig. 7. Obviously, the flow direction and the filling process of the oil have been changed when another inlet was introduced on the top of the outside part compared with Fig.2 and Fig.5. The oil firstly flows to the center part and the outside part via the two inlets and then diffuses down. From Fig.7 (a) and (b), it can be found that the oil in the center part is faster than that in the outside part to reach the bottle of the transformer so it can continuously spread around to fill the bottom of the outside part and soak into the oil papers. As a result, the air bubbles are mainly concentrated on the bottom of the model and the top of the oil-paper space and a few air bubbles are formed on the outside surface of the model as shown in Fig. 7 (c). However, the air bubbles originally concentrated on the top of the center part are disappeared. These results indicate that when the inlet is set on the top of the center part and the outside part, the distribution of the residual air bubbles are obviously changed.

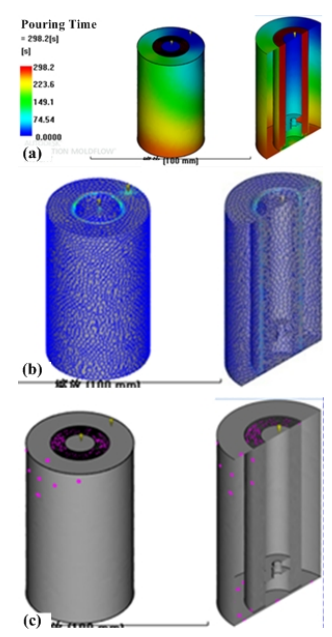

Fig.7 the simulating results of the transformer with two inlets set on the top of the center part and the outside part 
When the two oil inlets are set on the top of the outside part symmetrically, the related simulating results are displayed in Fig.8. In this situation, the oil firstly flows down to the bottom from the top of the outside part and then spread to the center part and the oil-paper spaces. Distinctly, the oil flow direction is changed as the distribution of the inlets adjusted compared with Fig.7. From the distribution of the air bubbles as shown in Fig.8 (c), it can be found there are few air bubbles formed on the top of the outside part and the air bubbles are mainly concentrate on the bottom of the model and the top of the oil-paper space. Moreover, a few air bubbles are generated on the top of the center part as there is no reserved pouring inlet.

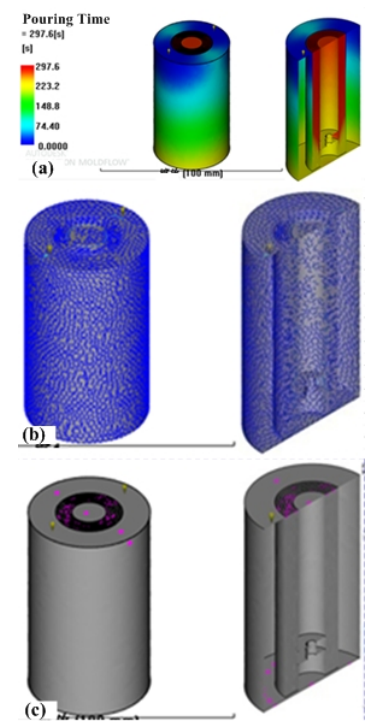

Fig. 8 The simulating results of the transformer with two inlets part symmetricallyset on the top of the outside

Based on the above analysis, the formation and distribution of the residual air bubbles are directly influenced by the oil flow direction and the air bubbles are always generated at the terminals, the inflexions and the rendezvous point of the oil flow as shown in the Fig.9. Furthermore, the flow direction and the soaking process are seriously affected by the oil inlets. When the oil inlets are set on the top of the transformer symmetrically, the inflexions and the rendezvous point of the oil flow will be rapidly decreased. Therefore, in order to exhaust the inside air of the transformer, the oil inlets should be designed properly and set on the top symmetrically.
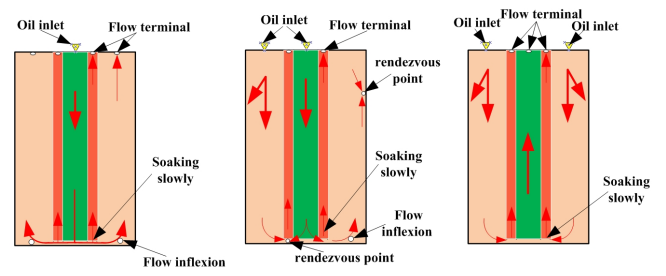

Fig. 9 the formation mechanism of the residual air bubbles during the pouring process for the oil-paper insulated transformer

\section{Conclusion}

The reliability of the oil-paper insulated transformer is seriously influenced by the vacuum degassing technology which is simulated by Moldflow. The results indicate that the filling speed and the residual air bubbles are affected by the space between the adjacent two oil papers. When the oil-paper space is increased, it is easier to fill the spaces in the oil papers and the pouring time is rapidly shortened. Moreover, when the oil papers are increased, the needed pouring pressure is 
raised and more residual air bubbles are located on the top of the oil-paper spaces. Besides, the direction, the soaking process and the formed air bubbles are directly impacted by the number and their distribution of the oil inlets. When the inlets are set symmetrically on the top of the 3D model, the residual air bubbles can be obviously decreased as the inflexions and the rendezvous point of the oil flow are rapidly reduced. This study can be applied to improve the reliability of the oil-paper transformer and it also can offer essential basis for its further research.

\section{References}

[1] Rui-jin Liao, Li-jun Yang, Jian Li, Stanislaw Grzybowski. Aging Condition Assessment of Transformer Oil-paper Insulation Model based on Partial Discharge Analysis. IEEE Transactions on Dielectrics and Electrical Insulation Vol. 18, No. 1; February 2011, 303-311

[2] Maik Koch, Thomas Prevost. Analysis of Dielectric Response Measurements for Condition Assessment of Oil-Paper Transformer Insulation. IEEE Transactions on Dielectrics and Electrical Insulation Vol. 19, No. 6; December 2012, 1908-1915

[3] Hamed Dashti and Majid Sanaye-Pasand. Power Transformer Protection Using a Multiregion Adaptive Differential Relay. IEEE TRANSACTIONS ON POWER DELIVERY, VOL. 29, NO. 2, APRIL 2014, 777-785

[4] S. Jayaram, "Effect of Streaming Potential on Heat Transfer in Transformer Oil", IEEE Trans. Dielectr. Electr. Insul., Vol. 3, pp. 410-416, 1996.

[5] I. A. Metwally, "Influence of Solid Insulation Phase on Streaming Electrification of Transformer Oil", IEEE Trans. Dielectr. Electr. Insul., Vol. 4, pp. 327-340, 1997.

[6] J. Yang, X. Chi, L. Ding and L. Ji, "Creeping Discharge Performance of Oil-Paper Insulation with Streaming Electrification", IEEE Trans. Dielectr. Electr. Insul., Vol. 4, pp. 780-784, 1997.

[7] distribution to electrical breakdown of insulating materials", IEEE Trans. Dielectr. Electr. Insul, Vol. 12, pp.11-16, 2005.

[8] R. Liao, S. Liang, L. Yang, J. Hao and J. Li, "Comparison of ageing results for transformer oil-paper insulation subjected to thermal ageing in mineral oil and ageing in retardant oil", IEEE Trans. Dielectr. Electr. Insul, Vol. 19, pp.225-232, 2012.

[9]P. Przybylek, H. Moranda, K. Walczak and H. Moscicka-Grzesiak. Can the Bubble Effect Occur in an Oil Impregnated Paper Bushing? IEEE Transactions on Dielectrics and Electrical Insulation Vol. 19, No. 6; December 2012, 1879-1883 